DOI: https://doi.org/10.11144/Javeriana.upsy16-2.ipip

\title{
Implementación del programa de intervención psicológica en resiliencia para cuidadores informales de pacientes con demencia tipo Alzheimer PIRCA*
}

\section{Implementation of the Psychological Intervention program in Resilience for Informal Caregivers of Patients with Alzheimer's Dementia PIRCA}

Recepción: Junio 19, 2014 | Aprobación: 24 Enero 2017

\author{
Ara Mercedes Cerquera Córdoba ${ }^{a}$ \\ Universidad Pontificia Bolivariana, Colombia \\ ORCID: http://orcid.org/0000-0002-6773-1495 \\ Daysy Katherine Pabón Poches \\ Universidad Pontificia Bolivariana, Colombia \\ Alexis Lorenzo Ruíz \\ Universidad de la Habana, Cuba
}

\begin{abstract}
a Autora de correspondencia. Correo electrónico: ara.cerquera@upb.edu.co
\end{abstract}

Para citar este artículo: Cerquera Córdoba, A. M., Pabón, D. K., \& Ruiz, A. L. (2017). Implementación del programa de intervención psicológica en resiliencia para cuidadores informales de pacientes con demencia tipo Alzheimer PIRCA. Universitas Psychologica, 16(2), 1-12. https://doi.org/10.11144/Ja veriana.upsy16-2.ipip

\section{RESUMEN}

Los programas de intervención psicológica de enfoque cognitivo conductual para cuidadores informales de pacientes con Alzheimer han sido hasta el momento los más utilizados por la efectividad de sus resultados. El objetivo de este estudio es la implementación del programa PIRCA (Programa de Intervención en Resiliencia para Cuidadores Informales de pacientes con Alzheimer) para observar los cambios presentados en los sujetos después de haber participado en el programa. Participaron 10 cuidadores informales, que no recibían remuneración, sin capacitación y convivían con el paciente. Se diseñó un estudio experimental transversal descriptivo comparativo, tipo pre-test/ post-test. Los resultados indicaron que los puntajes de las variables con mayor variabilidad fueron depresión estado, eutimia estado, sobrecarga, y estrategias de afrontamiento como búsqueda de apoyo social y religión. Con lo que se concluye que intervenir la resiliencia responde a una necesidad básica de los cuidadores ya que está presente en las características de personalidad del cuidador.

Palabras clave

programa de intervención; resiliencia; cuidador informal; Alzheimer

\section{ABSTRACT}

Psychological intervention programs of cognitive behavioral approach to informal caregivers of Alzheimer's patients have been so far, the most used by the effectiveness of their results. The objective of this study is the implementation of the program PIRCA (Resilience Intervention Program for Informal Caregivers of Alzheimer's patients) submitted to monitor changes in subjects after participating in the program. Participants were 10 informal caregivers, who were unpaid, untrained and lived with the patient. A cross-sectional comparative experimental study was designed as a pre-test/post-test type. The results indicated that the scores of the 
Ara Mercedes Cerquera Córdoba, Daysy Katherine Pabón Poches, Alexis Lorenzo Ruíz.

variables with depression which more variable state were: euthymic state, overload and coping strategies such as seeking social support and religion. Thus, it is concluded that intervene resilience responds to an own basic for caregivers as it is present in the personality characteristics of the caregiver.

Keywords

intervention program; resilience; informal caregiver; Alzheimer

\section{Introducción}

La enfermedad de Alzheimer es una enfermedad degenerativa de curso progresivo más frecuente en la población adulta mayor (Romano, Nissen, Del Huerto \& Parquet, 2007). Se estima que el número de personas con demencia a nivel mundial será de 65.7 millones en 2030 y 115.4 millones en 2050. En Colombia, para el 2020 se espera que 260000 personas mayores de 60 años padezcan la enfermedad del Alzheimer (Informe Mundial sobre el Alzheimer, 2009). Esta enfermedad desencadena una situación de discapacidad y dependencia (Durán, Valderrama, Uribe, González, \& Molina, 2010) haciendo que el paciente con demencia requiera de la ayuda de otros para poder desenvolverse en las actividades de la vida diaria (Ruíz-Robledillo \& Moya-Albiol, 2012). El familiar que asume el rol de cuidador informal es descrito como una persona que provee ayuda en el contexto doméstico (Aparicio, Díaz, Cuéllar, Fernández, \& De Tena, 2008; Lavretsky, 2005) generando en él una serie de cambios importantes en aspectos sociales y psicológicos y otros ajustes en su vida (Achury, Castaño, Gómez \& Guevara, 2011; Espín, 2012).

Ante las diferentes consecuencias que trae para el cuidador asumir este rol, se han empezado a crear programas de intervención para los mismos. Los programas que se han utilizado con más frecuencia han sido los de respiro, de autoayuda, psico-educativos y psicoterapéuticos (Losada et al., 2007), aunque la literatura reporta otras formas de intervención, como las de auto ayuda (Rabinovitz et al., 2006) individual y familiar (Mittelman, Roth, Clay \& Haley, 2007; Mittelman, 2003) de apoyo telefónico (Rabinovitz et al., 2006; Gallager-Thompson et al., 2007; Mittelman et al., 2007) intervenciones psico-educativas desde enfermería (SánchezPascual, Mouronte-Liz, \& Olazarán-Rodríguez, 2001; Gerdner, Buckwalter \& Reed, 2002), intervenciones psicosociales con técnicas de relajación (Hosaka \& Sugiyama, 2003) actividad física (Castro, Wilcox, O'Sullivan, Baumann, \& King, 2002) y desde el área de terapia ocupacional (Gitlin et al., 2008).

En cuanto a los resultados que han generado estos estudios, se ha evidenciado por medio de revisiones teóricas, que las intervenciones con efectos más significativos han sido las psicotepeuticas, especialmente las que han utilizado el enfoque cognitivo conductual (Losada, Montorio, Izal, \& Márquez, 2005; Zabalegui et al., 2008). El programa PIRCA de enfoque cognitivo conductual, toma como centro de intervención la resiliencia (Cerquera \& Pabón, 2015), término psicológico definido como una característica de personalidad positiva que permite la adaptación del individuo ante situaciones amenazantes (Wagnild \& Young, 1993); incluye los siguientes elementos: competencia personal la cual indica auto-confianza, independencia, decisión, invencibilidad, poderío, ingenio, y perseverancia; y aceptación de uno mismo y de la vida, la cual representa adaptabilidad, balance, flexibilidad y una perspectiva de vida estable, refleja aceptación por la vida y un sentimiento de paz, a pesar de la adversidad (Salgado, 2005).

Teniendo en cuenta los componentes de la resiliencia y los resultados obtenidos en el estudio de Cerquera \& Pabón (2015) donde la resiliencia correlacionó positivamente con el apoyo social, estrategias de afrontamiento como reevaluación positiva, solución de problemas y negativamente con espera, religión, búsqueda de apoyo profesional, se diseñó el programa PIRCA. A partir de estos resultados se plantea la implementación del programa para observar los cambios presentados en los cuidadores informales de pacientes con demencia tipo Alzheimer después de haber participado en el programa. 


\section{Método}

\section{Diseño}

Experimental transversal descriptivo comparativo, tipo pre-test/post-test.

\section{Participantes}

Este estudio contó con la participación de 10 cuidadores informales de pacientes con demencia tipo Alzheimer, participantes del programa de intervención en resiliencia para cuidadores, 9 mujeres y 1 hombre, de la ciudad de Bucaramanga, en edades comprendidas entre los 55 y 80 años y quienes llevaban cuidando a su familiar como mínimo 6 meses, 8 horas diarias, la enfermedad de Alzheimer estaba dentro de los tres estadíos y su familiar era madre, padre, esposa/esposo. Los participantes cumplían con los siguientes criterios de inclusión: no recibir remuneración por ser cuidador, ni tener ningún tipo de capacitación formal sobre la enfermedad, convivir en el mismo domicilio del enfermo con Alzheimer y haber estado al cuidado del mismo como mínimo 6 meses, 8 horas diarias.

\section{Procedimiento}

Fase I. Selección de instrumentos y revisión teórica:

Esta fase consistió en revisión de literatura pertinente para definir conceptos claves del proyecto, escoger los instrumentos más adecuados para la población y posteriormente contrastar resultados. Así mismo, se entrenó al grupo de investigación de Calidad de vida en la tercera edad de la Universidad Pontificia Bolivariana de Bucaramanga, Colombia, estudiantes tesistas de último año y jóvenes investigadoras de Colciencias, para la aplicación de las pruebas previstas y la entrevista semiestructurada.

Fase II. Contacto con la muestra

En esta fase se inició la búsqueda de instituciones de salud que trabajaran con adultos mayores y más específicamente con demencias.
Así, se presentó a dos instituciones la propuesta de trabajo para posteriormente acceder a una base de datos de pacientes con Alzheimer. Elaborada la base de datos, se procedió a llamar a cada uno de las casas de estos pacientes para establecer contacto con un familiar a cargo, presentar la investigación y el respectivo consentimiento informado en caso de aceptación en la participación del estudio.

Fase III. Aplicación pre test

Habiendo establecido contacto con la muestra, se procedió a verificar que cumplieran con los criterios de inclusión para finalmente concertar una cita donde recibiría la visita de los investigadores, quienes aplicarían las pruebas pre test para la evaluación del programa.

Fase IV. Ejecución de programa de intervención

Se procedió a invitar a 70 cuidadores que de forma aleatoria fueron seleccionados para la participación en el programa. Del total de cuidadores invitados iniciaron $30 \mathrm{y}$ culminaron 10 , esta fase se llevó a cabo en el Instituto de familia y vida de la Universidad Pontificia Bolivariana Bucaramanga, en el periodo comprendido de Febrero a Abril de 2014. La implementación del programa se desarrolló en diez sesiones. La primera sesión es de presentación, tiene como objetivo establecer el contrato terapéutico y crear expectativas frente al programa; la segunda sesión, este soy yo, cuyo propósito es mejorar la imagen que se tiene de sí mismo reconociendo emociones y facilitando la autoconfianza; la sesión tres; aceptándome, busca reconocer por medio de las experiencias positivas las características de vida estable; en la sesión cuatro, de apoyo social, busca identificar el tipo de apoyo social que tiene el cuidador para potenciar el balance personal, la quinta sesión, así me ven, pretendía fortalecer la autoestima del cuidador reconociendo las cualidades que otros ven en él; la sexta sesión, queriéndolo hacer y lo logré, tiene como objetivo reconocer la perseverancia y la decisión como competencias personales desarrolladas; la séptima, lo que sería capaz de hacer, la cual busca identificar la decisión como una característica importante de la competencia personal; la sesión ocho, reconociendo mis derechos y aprendiendo a pedir 
ayuda, busca identificar los derechos del cuidador para mejorar su calidad de vida; sesión nueve, mis poderes motivacionales, su objetivo es inhibir acciones, sentimientos creencias indeseables con el fin de que el cuidador visualice la aparición de logros y objetivos que lo lleven a desarrollar las competencias personas de resiliencia y la décima sesión, cierre, el objetivo es evaluar los resultados del programa de intervención.

Fase V: Evaluación de resultados

Terminada la ejecución del programa de intervención, se da paso a la evaluación de proceso por medio de tres cuestionarios con elaboración propia y la aplicación pos test dando paso a la calificación de las pruebas, tabulación en el sistema de datos SPSS. 22 para el respectivo análisis.

\section{Instrumento}

Programa de intervención psicológica en cuidadores informales de pacientes con demencia tipo Alzheimer PIRCA (Cerquera Eु Pabón, 2015)

El programa PIRCA, de enfoque cognitivo conductual, tiene como objetivo realizar una intervención psicológica en resiliencia a cuidadores informales de pacientes con demencia tipo Alzheimer, está conformado por 10 sesiones, cada una con una duración aproximada de 90 minutos.

Escala de Resiliencia de Wagnild y Young (1993)

La versión en idioma español adaptada fue tomada de Novella (2002). Consta de 25 ítems escritos en forma positiva con un sistema de respuesta tipo Likert que va de 1 a 7 puntos. Comprende dos dimensiones: competencia personal y aceptación de sí mismo y de la vida la validez de la escala es de 0.79 , según el alfa de Cronbach. A mayor puntuación, mayor nivel de resiliencia tanto en la escala total como en sus dimensiones (Quiceno, Vinacia, \& Remor, 2011).
Escala de carga del cuidador Zarit adaptada al castellano por Martín et al., (1996)

Fue diseñada para valorar la vivencia subjetiva de sobrecarga sentida por el cuidador principal de pacientes con demencia. Se trata de un instrumento autoaplicado de 22 ítems que explora los efectos negativos sobre el cuidador en distintas áreas de su vida: salud física, psíquica, actividades sociales y recursos económicos. Cada cuestión se evalúa mediante una escala tipo Likert con 5 posibles respuestas que van desde nunca a casi siempre y que puntúan entre 1 y 5 . La puntuación total es la suma de todos los ítems y el rango, por lo tanto oscila entre 22 y 110 . La consistencia interna arroja un coeficiente alfa de Cronbach de 0.91. La fiabilidad es de 0.86.

Escala de estrategias de Coping- modificada (EEC-M)

Modificada por Londoño et al. (2006). En esta escala se plantean 69 ítems que representan diferentes formas que emplean las personas para afrontar los problemas o situaciones estresantes que se les presentan en la vida. El sujeto debe señalar el número que mejor indique que tan habitual ha sido esta forma de comportamiento ante situaciones estresantes con los siguientes valores: 1 nunca, 2 casi nunca, 3 a veces, 4 frecuentemente, 5 casi siempre, 6 siempre. La consistencia interna arroja un coeficiente alfa de Cronbach de 0.847 .

\section{Cuestionario MOS de apoyo social}

Validado por Espíndola, Enrique y Carmelo (2005) versión argentina. El cuestionario centraliza el análisis en la percepción de ayuda. Consta de 20 ítems con una escala tipo likert expresada en los siguientes valores: 1 nunca, 2 pocas veces, 3 algunas veces, 4 la mayoría de veces, 5 siempre. Con 0.919 de consistencia interna según Alfa de Cronbach. 


\section{Consideraciones éticas}

Para la realización de esta investigación se tendrán en cuenta los lineamientos de la Ley 1090 de 2006, artículo 2 numeral 9, referido a la investigación con humanos y el respeto de la dignidad y el bienestar de las personas que participan con pleno conocimiento de la investigación. Igualmente, se asumió lo indicado en el artículo 50, el cual señala que las investigaciones realizadas por los profesionales de la psicología deben estar basadas de los principios éticos de respeto y dignidad, y salvaguardar el bienestar de los derechos delos participantes. Así mismo, la propuesta de investigación será sometida a revisión por el comité de ética de la Universidad Pontificia Bolivariana Bucaramanga.

\section{Resultados}

En el siguiente apartado se presentarán los criterios de evaluación de proceso y resultado en la implementación del programa de intervención en resiliencia PIRCA. Para la evaluación de proceso se elaboraron tres cuestionarios, un cuestionario que indica el nivel de satisfacción que experimentan los cuidadores con el programa al que participaron sesión por sesión. Dicho cuestionario es elaborado con escala tipo Liker con opciones de respuesta donde la máxima satisfacción tenía una valoración de cinco y la mínima de uno por cada ítem. Se observan los puntajes obtenidos en cada categoría y se obtiene la media correspondiente a cada factor por sesión.

\section{TABLA 1}

Evaluación de criterios de proceso implementación del programa y satisfacción

\begin{tabular}{llllll}
\hline Satisfacción con el contenido teórico & 1 & 2 & 3 & 4 & 5 \\
Satisfacción con el contenido príctico & 1 & 2 & 3 & 4 & 5 \\
Satisfacción con los terapeutas & 1 & 2 & 3 & 4 & 5 \\
Claridad de la información transmitida & 1 & 2 & 3 & 4 & 5 \\
Adecuación de los ejercicios & 1 & 2 & 3 & 4 & 5 \\
Adecuación de los materiales & 1 & 2 & 3 & 4 & 5 \\
Responde a las expectativas & 1 & 2 & 3 & 4 & 5 \\
Utilidad de lo aprendido & 1 & 2 & 3 & 4 & 5 \\
\hline
\end{tabular}

Fuente: elaboración propia.

El segundo cuestionario evaluó el cumplimiento y la calidad de las tareas asignadas al cuidador según cada sesión, teniendo en cuenta dos ítems; el primero evaluaba la realización de los ejercicios y actividades en casa y el segundo, la calidad de los ejercicios y actividades.

\section{TABLA 2}

Evaluación criterios de proceso por parte del terapeuta

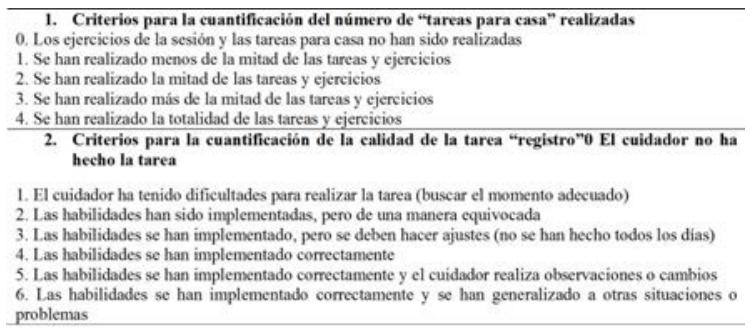
Fuente: elaboración propia.

El tercer cuestionario evaluó la satisfacción del cuidador con el programa de intervención por medio de 17 ítems y una lista de 11 frases incompletas.

\section{TABLA 3}

Cuestionario de satisfacción con el tratamiento

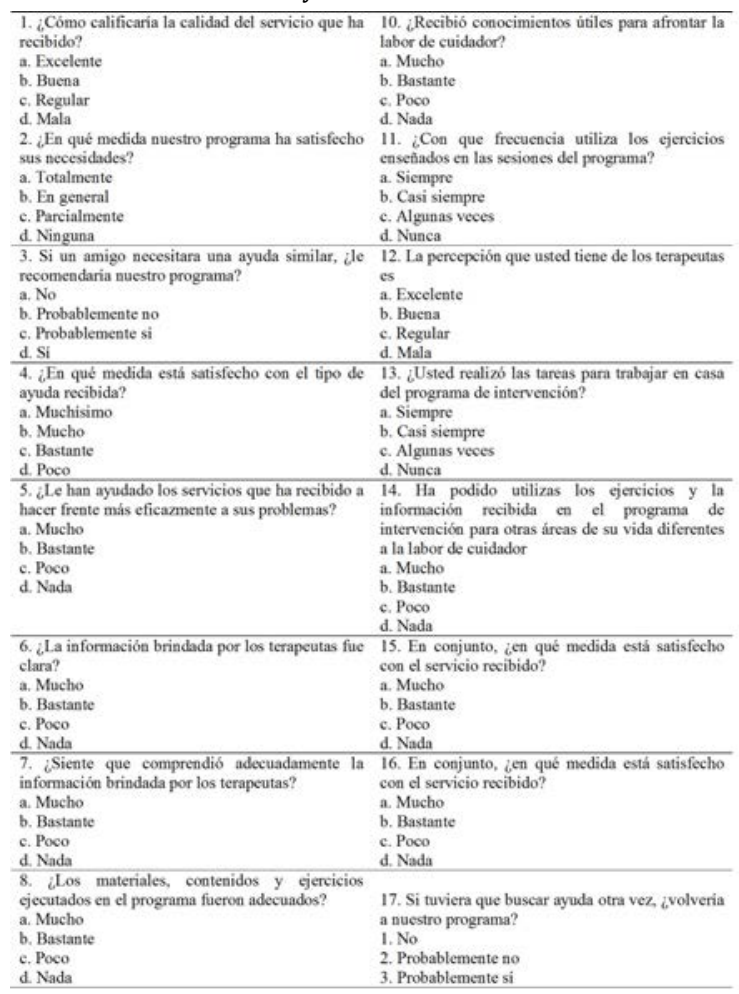

Fuente: elaboración propia. 
Para la evaluación de resultados, se aplica el pos-test y se comparan las medias obtenidas con el pre-test.

TABLA 4

Comparación de media depresión

\begin{tabular}{|c|c|c|c|c|c|c|c|}
\hline \multicolumn{2}{|c|}{ DEPRESIÓN } & $\begin{array}{l}\text { Puntuación } \\
\text { directa }\end{array}$ & Percentil & $\begin{array}{c}\text { Puntuación } \\
\text { directa }\end{array}$ & Percentil & $\begin{array}{c}\text { Puntuación } \\
\text { directa }\end{array}$ & Percentil \\
\hline \multirow{3}{*}{ ESTADO } & & Distimia & Distimia & Eutimia & Eutimia & Total & Total \\
\hline & PRE & 6.1 & 4.7 & 8.9 & 18.9 & 15.3 & 23.2 \\
\hline & POS & 5.9 & 5 & 7.5 & 8.6 & 12.8 & 11.2 \\
\hline \multirow{3}{*}{ RASGO } & & Distimia & Distimia & Eutimia & Eutimia & Total & Total \\
\hline & PRE & 6.1 & 3.4 & 7.6 & 8.2 & 13.9 & 8.7 \\
\hline & POS & 5.6 & 4.4 & 6.8 & 6.8 & 12.1 & 6.7 \\
\hline
\end{tabular}

Fuente: elaboración propia.

En la tabla 4 se puede observar que los puntajes del post test disminuyeron considerablemente especialmente en depresión estado.

\section{TABLA 5}

Comparación de medias apoyo social, carga y resiliencia

\begin{tabular}{lll}
\hline Variable & PRE & POS \\
\hline Emocional & 28.4 & 30.1 \\
Ayuda Material & 15.6 & 14.4 \\
Interacción social & 14.3 & 14.6 \\
Afectivo & 11.6 & 11.9 \\
Global & 71.8 & 71 \\
Zarit & 50 & 37.3 \\
Competencia personal & 104.2 & 102.9 \\
Aceptación de sí mismo de la vida & 46.9 & 46.4 \\
Resiliencia & 151.2 & 149.2 \\
\hline
\end{tabular}

Fuente: elaboración propia.

En la tabla 5 se observa que la percepción de apoyo material disminuyó teniendo en cuenta que los cuidadores al desplazarse al programa requerían de un mayor apoyo por parte de los familiares para cuidar al paciente. El apoyo emocional incrementó, lo que indica que el participar del programa los hace percibir un apoyo emocional. Los otros puntajes de la escala MOS se mantuvieron o aumentaron de forma mínima, queriendo con ello apoyar el impacto de la intervención psicológica en los cuidadores. En la prueba Zarit se observa una disminución importante que muestra la efectividad del programa para disminuir la carga, mientras que en resiliencia no hay variaciones importantes.
TABLA 6

Estrategias de afrontamiento pre test y pos test

\begin{tabular}{ccccccc}
\hline ESTRATEGLA & $\begin{array}{c}\text { Solución } \\
\text { problemas }\end{array}$ & $\begin{array}{c}\text { Búsqueda } \\
\text { apoyo } \\
\text { social }\end{array}$ & Espera & Religión & $\begin{array}{c}\text { Evitación } \\
\text { emocional }\end{array}$ & $\begin{array}{c}\text { Apoyo } \\
\text { profesional }\end{array}$ \\
\hline PRE & 38.1 & 29.6 & 21.9 & 34.5 & 17.3 & 15 \\
POS & 38.3 & 23.9 & 21.7 & 24.5 & 15.9 & 14.9 \\
\hline ESTRATEGIA & Reacción & Evitación & Reevaluadi & Expresión & Negación & Autonomia \\
\hline agresiva & cognitiva & ón positiva & dificultad & & \\
\hline PRE & 8.8 & 13.8 & 21.6 & 9.1 & 7.5 & 5.4 \\
POS & 8.5 & 14.1 & 21.8 & 8.7 & 7.8 & 4.4 \\
\hline \multicolumn{7}{c}{ Fuente: elaboración propia. }
\end{tabular}

En la tabla 6 se observan los cambios referidos a las puntuaciones pos test en donde se determinan variaciones importantes en las estrategias búsqueda de apoyo social, evitación emocional, expresión de la dificultad, e incrementando la evitación cognitiva.

\section{Discusión y conclusiones}

El programa de intervención aborda una problemática importante que afecta a una población vulnerable, los cuidadores informales de pacientes con Alzheimer. Según Cerquera \& Pabón (2015) dicho programa fue diseñado para ejecutar en 10 sesiones cada una de las cuales presentaba un propósito específico, con técnicas adecuadas a dicho propósito y al enfoque acogido, tal como lo plantean las autores del programa.

En la evaluación de resultados del programa de intervención PIRCA se encuentra que los cuidadores presentan resiliencia en la aplicación del pre y post test, esta se evidencia cuando las respuestas están en la media de 144.16 en total. En las subescalas de aceptación de sí mismo y de la vida 45.70 y en competencias personales 98.4. Los datos arrojados son relevantes en la medida que para ser cuidador se debe ser resiliente. Esto lo confirma la literatura en la definición de resiliencia, entendida como "una trayectoria estable de funcionamiento saludable a lo largo del tiempo, así como la capacidad para generar experiencias y emociones positivas" (Bonanno, 2004). Para la muestra estudiada, las mediciones en resiliencia se mantuvieron, lo que nos indica que esta es una característica personológica que determina las particularidades de un individuo, coincidente con la explicación del Modelo de características psicobiológicas y genética 
molecular de las personas adultas resilientes, el cual confirma que las emociones positivas como el optimismo y el sentido del humor, elementos trabajados desde el programa de intervención, posibilitan la regulación de la actividad autonómica y se fortalecen los circuitos de recompensa, lo que llevaría a actuar de manera resiliente (Quiceno \& Vinaccia, 2010).

Según Saavedra y Villalta (2008), la resiliencia es un rasgo personal cultivado a lo largo de la historia del sujeto, y que posiblemente, se ha constituido como vínculo temprano, y que es condición para el desarrollo de una particular apropiación de los sucesos de la vida. Es decir, aquello que el sujeto tiene como rasgo distintivo ha sido aprendido en relación a otros, y al ser aprendido puede cambiar, o está en constante proceso de interpretación. En el caso de cuidar a un enfermo de Alzheimer, situación importante en la vida, el cuidador debe recurrir a la resiliencia para poder asumir el rol de cuidador.

En cuanto a los resultados de la depresión, evaluados por medio de la escala IDER, en el pre test no se evidenció presencia de sintomatología depresiva, aunque los puntajes de depresión estado eran mayores que los puntajes de depresión rasgo. En el pos test es de anotar que distimia tanto estado como rasgo incrementaron, resultados coherentes con la literatura en que se afirma que la depresión es una variable difícil de intervenir en los cuidadores informales puesto que la sintomatología tiende a incrementar por las características propias del rol (Espín, 2012). Por otro lado, la Eutimia tanto en rasgo como en estado disminuyeron, lo cual no es coincidente con (Fernández-Lansac \& Crespo, 2011) quien afirma que la depresión irá incrementando a medida que el avance de la enfermedad del paciente que cuida avanza. Teniendo en cuenta el programa PIRCA, se puede afirmar que los cuidadores resilientes excluyen los síntomas depresivos, por lo que el programa muestra que podría influir sobre esta variable siempre y cuando se presente la sintomatología depresiva.

Dentro de otros elementos a destacar por tener relación directa con el enfoque del programa, cabe mencionar aquellos puntajes que incrementaron en las estrategias de afrontamiento utilizadas por los sujetos de la muestra; entre ellas se encuentra, la evitación cognitiva, definida como "una estrategia cognitiva en la que se busca eliminar o neutralizar los pensamientos valorados como negativos $\mathrm{O}$ perturbadores a través de la distracción o negación” (Londoño et al., 2006); la solución de problemas, descrita como "la secuencia de acciones orientadas a solucionar el problema teniendo en cuenta el momento oportuno para analizarlo o intervenirlo" (Londoño et al., 2006), de acuerdo con las estrategias implementadas en el programa PIRCA, les permitió a los sujetos identificar aquellas problemáticas que les resultaban más complejas de abordar y darles solución en los momentos oportunos, esto trabajado específicamente en la sesión No. 6 "queriéndolo hacer y lo logré". Otros resultados importantes en relación a las estrategias de afrontamiento es la búsqueda de apoyo social, en la que los puntajes disminuyeron, con lo que se puede hipotetizar que los sujetos encontraron una utilidad en el programa y no requieren de la utilización de esta estrategia en la misma medida que antes. Así mismo, la estrategia de espera disminuyó su puntuación, lo que determina entonces que los sujetos empezaron a tomar una posición activa frente al problema.

En relación a la resiliencia, la estrategia de Reevaluación positiva incrementó en su utilización. Londoño et al. (2006) la define como "una estrategia cognitiva que busca aprender de las dificultades identificando los aspectos positivos del problema, es una estrategia de optimismo que contribuye a tolerar la problemática y a generar pensamientos que favorecen, al enfrentar la situación”. Esta relación es importante teniendo en cuenta que una de las características por medio de las cuales se define la resiliencia es el sentido del humor y la felicidad (Bonnano, 2004). Búsqueda de apoyo profesional, disminuyó, esta estrategia comportamental está en función de la búsqueda de recurso profesional para solucionar el problema o las consecuencias del mismo (Londoño et al., 2006). En cuanto a la utilización de esta estrategia está relacionada con las herramientas dadas en el programa que 
Ara Mercedes Cerquera Córdoba, Daysy Katherine Pabón Poches, Alexis Lorenzo Ruíz.

facilitan el adecuado afrontamiento a la situación problema sin requerir de ayuda terapéutica.

La estrategia de la religión disminuyó, los cuidadores del programa buscaron otras estrategias diferentes a las señaladas inicialmente. El rezo y la oración se consideran como una estrategia para tolerar el problema o para generar soluciones ante el mismo (Londoño et al., 2006). Sin embargo, se debe tener mucha precisión al analizar este dato, ya que el rezo y la oración son conceptos diferentes que hoy en día los sujetos utilizan, además es importante resaltar las diferencias en la pluralidad de cultos en la sociedad actual (Parker, 2008). Los resultados de la variable sobrecarga indican una disminución en la escala del cuidador Zarit, en la que se obtuvo un puntaje menor en el pos test, lo que indica que el programa afecta de manera importante esta variable. El trabajo grupal con los cuidadores permitió que la experiencia de cuidado se compartiera y que, según los testimonios de cada uno de ellos, no sintieran que solo ellos tenían que afrontar dicha situación.

Cabe resaltar que dentro de las estrategias de afrontamiento como otros modelos, se considera el afrontamiento activo frente a la búsqueda de soluciones y control emocional, además, se encuentran la flexibilidad cognitiva, reevaluación cognitiva y aceptación, como logro de comportamientos resilientes, (Quiceno et al., 2011), características encontradas en los cuidadores de la muestra. En cuanto a las diferencias de medias entre el pre y post test cabe destacarse que en la mayoría de los puntajes de las variables el cambio no es mayor, lo que está de acuerdo con Knight, Lutzky \& MacofskyUrban, 1993; Brodaty et al., 2003; Pinquart \& Sörensen, 2006), al referirse al tamaño del efecto de las intervenciones con cuidadores que es generalmente pequeño y tiende a ser menor en fases de seguimiento. La dificultad para obtener efectos positivos a través de las intervenciones no quiere decir que no hayan tenido lugar avances notables en la investigación en el área, lo que es evidente en el cambio en los puntajes de las variables depresión estado, eutimia estado, sobrecarga, y estrategias de afrontamiento como búsqueda de apoyo social y religión, en el programa PIRCA.

Así, existen datos que permiten concluir que no todas las intervenciones presentan el mismo grado de eficacia, las basadas en los modelos cognitivos y/o conductuales (Beck et al., 1979; Lewinsohn et al., 1978), de los cuales existen adaptaciones al cuidado (Knight et al., 2000; Losada et al., 2005), son las que permiten mayores beneficios, especialmente cuando se exige una participación activa por parte de los cuidadores (Gallagher-Thompson \& Coon, en prensa; Pinquart \& Sörensen, 2006).

En el caso del programa de intervención PIRCA, las tareas que se le colocan al cuidador deben ser respondidas para corroborar la implicación personal en el programa. Son precisamente este tipo de intervenciones las que han pasado de ser clasificadas, según los criterios de la División 12 de Psicología Clínica de la Asociación Americana de Psicología (Chambless \& Hollon, 1998, Chambless et al., 1998), como intervenciones probablemente eficaces (Gatz et al., 1998), en la actualidad, ser consideradas como intervenciones bien establecidas o empíricamente validadas.

En lo que refiere a intervenciones con cuidadores familiares o informales se halla coincidencia entre varios autores al concluir que las intervenciones psico-educativas o informativas no han sido suficientes para mejorar la calidad de vida del cuidador (Espín, 2008; Losada et al., 2005) y que por el contrario la mejor forma de intervenir en esta población es por medio de la intervención psicológica (Zabalegui et al., 2008; OrozcoGómez, Eustache-Rodriguez, \& Grosso-Torres, 2012; Losada et al., 2005).

Sin embargo, aun cuando se logra diseñar y ejecutar una intervención psicológica como PIRCA, y adicionalmente, se logran comprobar sus efectos de forma empírica, se pueden presentar limitaciones como la posibilidad de que los cuidadores no asistan rigurosamente a las sesiones; este estudio inició con 30 pacientes y finalizó con 10, lo que fue el mayor obstáculo para la implementación; los cuidadores generalmente no cuentan con disponibilidad de tiempo y/o 
soporte para asistir a las sesiones. Lo anterior, abre nuevas líneas de investigación en programas de respiro para cuidadores que estén orientados a mitigar estas limitaciones.

\section{Agradecimientos}

Implementación del programa de intervención PIRCA. Entidad financiadora: Dirección General de Investigaciones Universidad Pontificia Bolivariana Bucaramanga y COLCIENCIAS. Código: 090-0613-3100.

\section{Referencias}

Achury, D., Castaño, H., Gómez, L. \& Guevara, N. (2011). Calidad de vida de los cuidadores de pacientes con enfermedades crónicas con parcial dependencia. Investigación en Enfermeria: imagen y desarrollo, 13(1), 27-46.

Aparicio, M., Díaz, J., Cuéllar, B., Fernández, M., \& De Tena, A. (2008). La Salud Física y Psicológica de las Personas Cuidadoras: Comparación entre Cuidadores Formales e Informales. (Tesis para optar el título de doctor). Universidad Complutense de Madrid. España.

Beck, A. T., Rush, J. A. \& Shaw, B. F., (1979) Cognitive Therapy of Depression. New York: Guilford Press.

Bonanno, G. (2004). Loss, trauma, and human resilience: Have we underestimated the human capacity to thrive after extremely aversive events? American Psychologist, 59(1), 20-28.

Brodaty, H., Green, A. \& Koschera, A. (2003). Meta-analysis of psychosocial interventions for caregivers of people with dementia. Journal of the American Geriatrics Society, 51(5), 657-664.

Castro, C.M., Wilcox, S., O'Sullivan, P., Baumann, K., \& King, A.C. (2002). An exercise program for women who are caring for relatives with dementia. Psychosom Med, 64(3), 458-468.
Cerquera \& Pabón-Córdoba, A.M. \& PabónPoches, D.K. (2015). Modelo de intervención psicológica en resiliencia para cuidadores informales de pacientes con Alzheimer. Diversitas: Perspectiva Psicológica, 11 (2), 181-192. doi: https://doi.org/10.153 32/s1794-9998.2015.0002.01

Chambless, D.L, \& Hollon S. D. 1998. Defining empirically supported therapies. J. Consult Clinical Psycholy. 66(1), 7-18

Chambless, D.L, Sanderson, W.C, Shoham, V, Bennett Johnson, S., \& Pope, K.S. (1996). An update on empirically validated therapies. Clinical Psycholy, 49(2), 5-18

Durán, A., Valderrama, L., Uribe, A., González, A., \& Molina, M. (2010). Enfermedad crónica en adultos mayores. Revista universitas médica, 51 (1), 16-28.

Espín, A. (2008). Caracterización psicosocial de cuidadores informales de adultos mayores con demencia. Revista Cubana salud Pública, $34(3), 1-13$.

Espín, A. (2012). Factores de riesgo de carga en cuidadores informales de adultos mayores con demencia. Revista cubana de salud pública, 38(3), 393-402.

Espíndola, R., Enrique, S., \& Carmelo, H. (2005). Validación argentina del cuestionario MOS de apoyo social percibido. Psicodebate, 7 , 155-168.

Fernández-Lanzac, V., \& Crespo, M. (2011). Resiliencia, personalidad resiliente $\mathrm{y}$ crecimiento en cuidadores de personas con demencia en el entorno familiar: Una revisión. Clinica y salud, 22(1), 21-40.

Gallagher-Thompson, D. \& Coon, D.W. (en prensa). Evidence-based psychological treatments for distress in family caregivers of older adults. Psychology $\mathbb{E}$ Aging.

Gallager-Thompson, D., Gray, H., Tang, P., Pu, C., Leung, L., \& Wang, P., (2007). Impact of in-home behavioral management versus telephone support to reduce depressive symptoms and perceived stress in Chinese caregivers: results of a pilot study. American Journal of Geriatric Psychiatry, 15(5), 425-434. 
Gatz, M., Fiske, A., Fox., L.S., Kaskie, B., Kasl-Godley, J.E., McCallum, T.J., \& Wetherell, J.L. (1998). Empirically validated psychological treatments for older adults. Journal of Mental Health and Aging, 4(1), 9-46.

Gerdner, L., Buckwalter, K., \& Reed, D. (2002). Impact of a psychoeducational intervention on caregiver response to behavioral problems. Nursing Research, $51(6), 363-374$.

Gitlin, L., Winter, L., Burke, J., Chernett, N., Dennis, M., \& Hauck, W. (2008). Tailored activities to manage neuropsychiatric behaviors in persons with dementia and reduce caregiver burden: a randomized pilot study. American Journal of Geriatric Psychiatry, 16(3), 229-239.

Hosaka, T., \& Sugiyama, Y. (2003). Structured intervention in the family caregivers of the demented elderly and changes in their immune function. Psychiatry and Clinical Neurosciences, 57(2), 147-151.

Informe mundial sobre Alzheimer (2009). Resumen ejecutivo. Recuperado de http://www.alz.co.uk/research/files/Wor ldAlzheimerReport-Espanol.pdf

Knight, B.G., Lutzky, S.M., \& MacofskyUrban, F. (1993). A Meta-analityc review of interventions for caregiver distress: recomendations for future research. The Gerontologist, 33, 240-248

Knight, B.G., Silverstein, M., McCallum, T.J., \& Fox, L.S. (2000). A sociocultural stress and coping model for mental health outcomes among African American caregivers in Southern California. Journal of Gerontology: Psychological Sciences, 55(3), 142-150.

Lavretsky, H. (2005). Stress and depression in informal family caregivers of patients with Alzheimer's disease. Aging Health, 1(1), 117-133.

Lewinsohn, P. M., Muñoz, R. F., Youngren, M. A., \& Zeiss, A. M. (1978). Control your depression. Englewood Cliffs, NJ: PrenticeHall disfuncionales. Madrid: IMSERSO.

Losada, A, Montorio, I, Izal, M., \& Márquez, M. (2005). Estudio e intervención sobre el malestar psicológico de los cuidadores de personas con demencia. El papel de los pensamientos disfuncionales. Madrid: IMSERSO.

Losada, A., Márquez-Gonzalez, M., Peñacoba, C., Gallagher-Thompson, D. \& Knight, B. (2007). Reflexiones en torno a la atención a los cuidadores informales de personas con demencia y propuesta de una intervención interdisciplinar. Psicología Conductual, 15(1), 5776.

Londoño, H., Henao, G., Puerta, I., Posada. S., Arango, D. \& Acevedo, C. (2006). Propiedades psicométricas y validación de la escala de estrategias de coping modificada (eec-m) en una muestra colombiana. Universitas Psychologica, 5(2), 327-349.

Martin, M., Salvadó, I., Nadal, S., Mji, L., Rico, L., Lanz, J... \& Taussing, M. (1996). Adaptación para nuestro medio de ka escala de sobrecarga del cuidador. Revista de gerontología, 6(4), 338-345.

Mittelman, M. (2003). Community caregiving. Alzheimer's Care Quarterly, 4(4), 273 -285.

Mittelman, M., Roth, D., Clay, O., \& Haley, W. (2007). Preserving health of Alzheimer caregivers: impact of a spouse caregiver intervention. American Association for Geriatric Psychiatry, 15(9), 780-788.

Novella, A. (2002). Incremento de la resiliencia luego de la aplicación de un programa de psicoterapia breve en madres adolescentes. (Tesis de maestria). Universidad Nacional Mayor de San Marcos, Lima.

Orozco-Gómez, A., Eustache-Rodriguez, V., \& Grosso-Torres, L. (2012). Programa de intervención cognoscitivo conductual en la calidad de sueño de cuidadores familiares. Revista Colombiana de Enfermería, 7(7), 75-85.

Parker, C. (2008) Pluralismo religioso, educación y ciudadanía. Sociedad e estado, 23(2), 281-353.

Pinquart, M. \& Sörensen, S. (2006). Gender differences in caregiver stressors, social resources, and heath: an update meta- 
analysis. Journal of Gerontology, 61(1), 33-45.

Quiceno, J., \& Vinaccia, S. (2010). Resiliencia: una perspectiva de la enfermedad crónica en la población adulta. Pensamiento psicológico, 9(17), 69-82.

Quiceno, J., Vinaccia, S., \& Remor, E. (2011). Programa para potenciación de la resiliencia para pacientes con artritis reumatoide. Revista de Psicopatología y Psicología Clínica, 16(1), 27-47.

Rabinovitz, Y., Mausbach, B., Coon, D., Depp, C., Thompson, L. \& Gallagher-Thompson, D. (2006). The moderating effect of selfefficacy on intervention response in women family caregivers of older adults with dementia. American Journal of Geriatric Psychiatry, 14(8), 642-649.

Romano, M., Nissen, M, Del Huerto, N. \& Parquet, C. (2007). Enfermedad de Alzheimer. Revista de posgrado de la vía cátedra de medicina, 175, 9-12. Recuperado de http://med.unne.edu.ar/revista/revista1 75/3_175.pdf

Ruíz-Robledillo, N. \& Moya-Albiol, L. (2012). El cuidador: una visión actual. Revista de motivación y emoción, 1(22), 22-33.

Saavedra, E., \& Villalta, M. (2008). Medición de las características resilientes, un estudio comparativo en personas entre 15 y 65 años. Liberabit. Revista de Psicología, 14, 31-40.

Salgado, A. (2005). La ciencia y su respuesta frente la adversidad: estudios desde la perspectiva de la resilencia. Cultura, 19(19), 394-416

Sánchez-Pascual, P., Mouronte-Liz, P., \& Olazarán-Rodríguez, J. (2001). Beneficios de un programa de formación del cuidador en la demencia: experiencia piloto desde la enfermería especializada. Revista de Neurología, 33(5), 410-424.

Wagnild, G.M. \& Young, H.M. (1993) Development and psychometric evaluation of the Resilience Scale. Journal of Nursing Measurement. 1(2),165-178.

Zabalegui, A., Monserrat, D., Cabrera, E., Fernández-Puebla, A., Bardallo, D.,...
Remón, A. (2008). Eficacia de las intervenciones dirigidas a cuidadores principales de personas dependientes mayores de 65 años. Una revisión sistémica. Revista Española de Geriatría y Gerontología. 43(3), 157-166.

\section{ANEXO A}

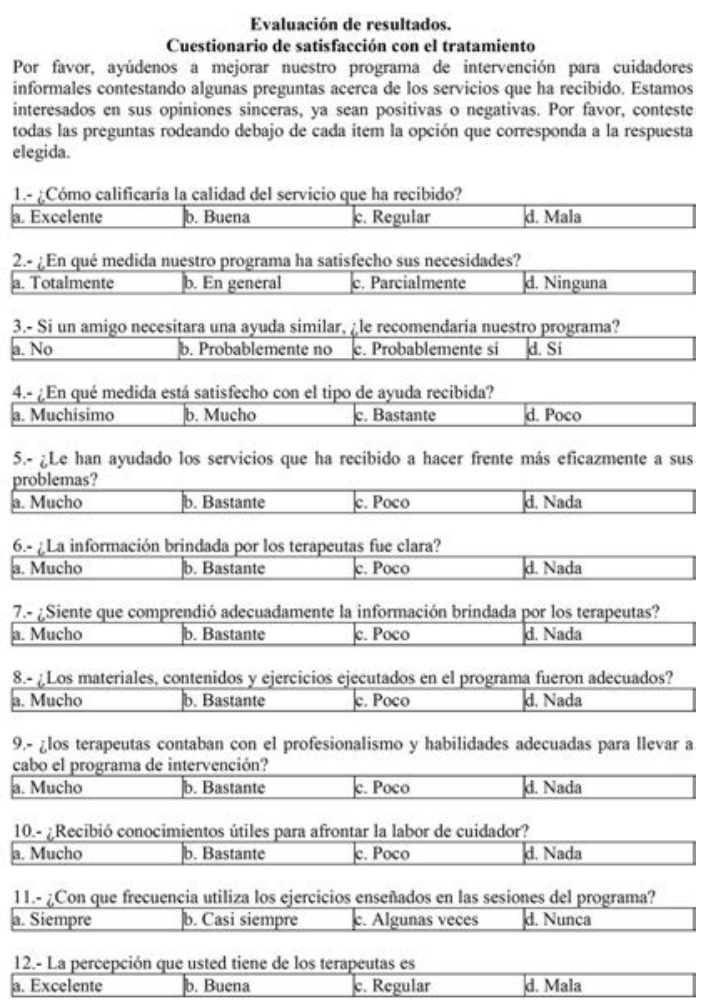


Ara Mercedes Cerquera Córdoba, Daysy Katherine Pabón Poches, Alexis Lorenzo Ruíz.

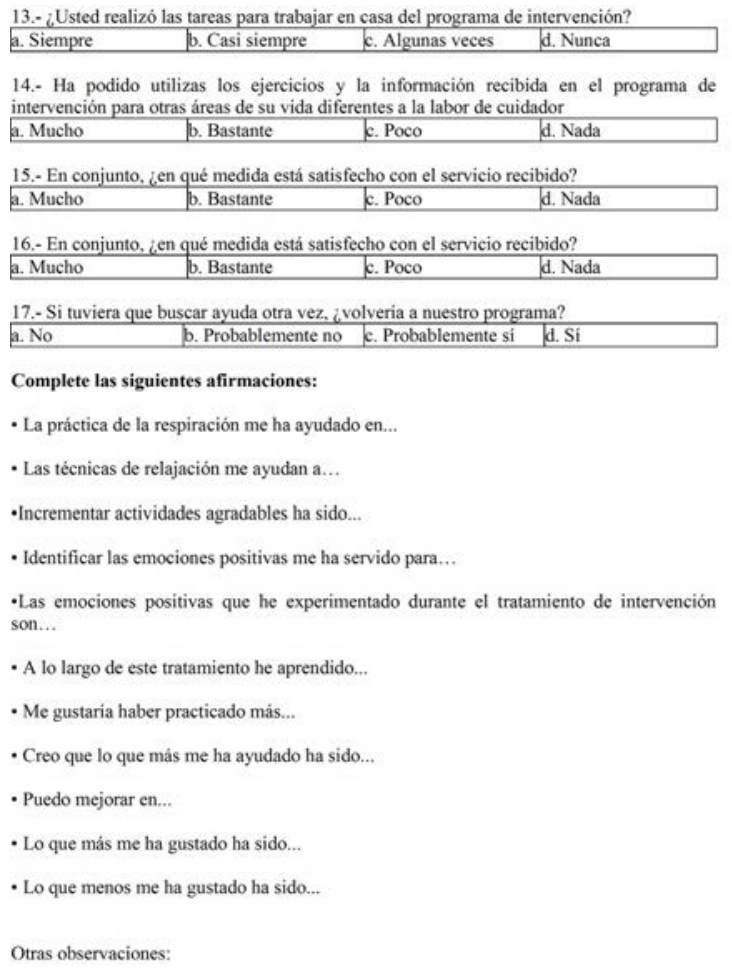

\section{Notas}

* Artículo de investigación 